\title{
Bonfils intubation in parallel with a supraglottic air-Q® intubating laryngeal airway
}

\author{
Sophie Drolet, MD · Sébastien Michaud, MD
}

Received: 27 September 2015/Revised: 1 October 2015/Accepted: 20 November 2015/Published online: 30 November 2015

(C) Canadian Anesthesiologists' Society 2015

\section{To the Editor,}

This report describes a novel method for exchanging a supraglottic laryngeal airway (SLA) for an endotracheal tube (ETT) in a pediatric patient with a challenging airway.

Three different methods can facilitate this exchange. The first method involves performing endotracheal intubation independently of the SLA-i.e., performing the intubation after removing the supraglottic device, such as an $\mathrm{LMA}^{\mathrm{TM}}\left(\right.$ Teleflex$^{\circledR}$, Morrisville, NC, USA) or a similar device. The second method involves performing the endotracheal intubation in series with the SLA-i.e., the airway stays in place and acts as a conduit to the glottis for the ETT. The third method involves performing the endotracheal intubation in parallel with the supraglottic airway-i.e., the anesthesia breathing circuit remains connected to the SLA while endotracheal intubation proceeds alongside with a device such as a Bonfils intubation fiberscope (Karl Storz $\mathrm{GmbH} \&$ Co. KG, Tuttlingen, Germany).

We recently used this parallel technique for airway management of a three-year-old boy scheduled for a bronchoscopy followed by an esophagogastroscopy. The child's parents gave their consent for performing the technique, taking photographs, and publishing this correspondence. The patient's medical history was significant for a prior tracheoesophageal fistula repaired in infancy, severe tracheomalacia, sleep apnea, asthma, and avascular left hip necrosis due to repeated high doses of

S. Drolet, MD $(\bowtie) \cdot S$. Michaud, MD

Department of Anesthesiology, CIUSSS de l'Estrie - Centre

Hospitalier Universitaire de Sherbrooke (CHUS), Sherbrooke,

QC, Canada

e-mail: sophie.drolet@usherbrooke.ca steroids given during multiple episodes of life-threatening respiratory events.

After applying standard monitors ${ }^{1}$ and induction of anesthesia with sevoflurane and propofol, a \#2 air-Q SLA (Mercury Medical, Clearwater, FL, USA) was easily inserted. The pediatrician performed flexible bronchoscopy through a swivel adaptor connected to the air-Q and topicalized the airway with lidocaine while the patient was spontaneously breathing sevoflurane. A Bonfils intubation fiberscope with a $3.5-\mathrm{mm}$ outer diameter was then mounted with a 4-mm cuffed ETT. The fresh gas flow was pre-emptively increased to $3 \mathrm{~L} \cdot \mathrm{min}^{-1}$ to compensate for possible circuit leaks. The Bonfils scope was then inserted into the pharynx beside the SLA using a retromolar approach. After passing around the right side of the air-Q's inflated cuff, the glottic opening was easily identified in the midline. The pre-loaded ETT was then advanced through the vocal cords, the ETT cuff was inflated, and the breathing circuit was transferred from the air-Q to the ETT without any change in the patient's oxygen saturation (Figure).

We can compare the three different ETT exchange methods described above according to the type of ETT used, the ease of removing the laryngeal airway device, the ease of intubation, the duration of required apnea during the exchange, the potential for gas leaks, and the overall ease at maintaining the patients' well-being. The independent and parallel methods facilitate placement of a correctly sized cuffed ETT on the first attempt, and both methods allow easy removal of the SLA. The in series method provides a direct path to the glottis through the SLA's breathing tube. ${ }^{2}$ In the parallel method, the midline of the laryngeal airway cuff can serve as a visual guide for advancing the Bonfils towards the glottis. Nevertheless, the parallel technique can increase gas leaks, which could possibly lead to inadequate ventilation. This did not occur 


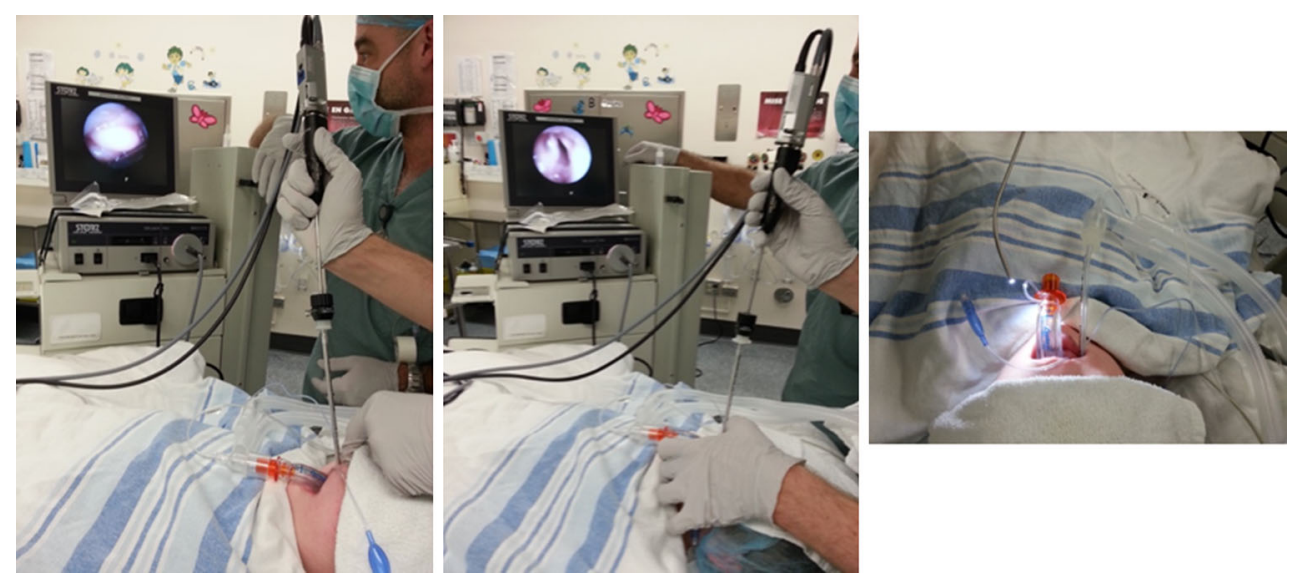

Figure The sequential images outline three steps for performing intubation with a Bonfils fiberscope while an air- ${ }^{\circledR}$ intubating laryngeal airway remains connected to the breathing circuit. The left image shows a Bonfils fiberscope mounted with a cuffed endotracheal tube (ETT) and inserted using a retromolar approach. In the centre

in our case because we had increased the fresh gas flow in advance. With the independent and in series methods, transient disconnection from the breathing circuit could potentially result in both desaturation and lightening the depth of anesthesia. ${ }^{3-5}$ A resulting reduction in anesthesia depth could increase the risk of bronchospasm, which in turn could further hasten the onset of hypoxemia. The parallel technique is the only method that provides continuous oxygenation, ventilation, and administration of volatile anesthetics to facilitate performing intubation in a safe manner without any time pressure.

We concluded that the use of Bonfils intubation in parallel with a SLA should be a consideration when planning an ETT exchange technique.

Acknowledgements The author sincerely thanks M. Dany Sawyer for his technical support, and Drs Johanne Carrier and Marie-José Colas for reviewing the correspondence.

Conflicts of interest None declared. image, the Bonfils fiberscope passes around the right side of the inflated cuff of the air-Q. The right image shows that the pre-loaded ETT is advanced through the vocal cords, its cuff is inflated, and the breathing circuit is transferred from the supraglottic laryngeal airway to the ETT

Funding None.

\section{References}

1. Merchant $R$, Chartrand D, Dain $S$, et al. Guidelines to the practice of anesthesia-revised edition 2015. Can J Anesth 2015; 62: 54-67.

2. Jagannathan $N$, Kozlowski RJ, Sohn LE, et al. A clinical evaluation of the intubating laryngeal airway as a conduit for tracheal intubation in children. Anesth Analg 2011; 112: 176-82.

3. de Graaff JC, Bijker JB, Kappen TH, van Wolfswinkel L, Zuithoff $N P$, Kalkman CJ. Incidence of intraoperative hypoxemia in children in relation to age. Anesth Analg 2013; 117: 169-75.

4. Hardman JG, Wills JS. The development of hypoxaemia during apnoea in children: a computational modelling investigation. $\mathrm{Br} \mathrm{J}$ Anaesth 2006; 97: 564-70.

5. Eich C, Timmermann A, Russo SG, et al. A controlled rapidsequence induction technique for infants may reduce unsafe actions and stress. Acta Anaesthesiol Scand 2009; 53: 1167-72. 